\title{
THE EFFECT OF VARIABLE CURRENTS ON INTERNAL SOLITARY WAVES
}

\author{
X. ZHOU \\ Institute of Mechanics, Chinese Academy of Sciences, Beijing (People's Republic of China)
}

\section{R. GRIMSHAW}

School of Mathematics, University of N.S.W., Kensington, P.O. Box 1, N.S.W. 2033 (Australia)

(Received December 17, 1987; revised June 9, 1988; accepted August 26, 1988)

\section{ABSTRACT}

Zhou, X. and Grimshaw, R., 1989. The effect of variable currents on internal solitary waves. Dyn. Atmos. Oceans, 14: 17-39.

The effect of variable currents on internal solitary waves is described within the context of a variable coefficient Korteweg-de Vries (KdV) equation, and the approximate slowly varying, solitary-wave solution of this equation. The general theory which leads to the variable coefficient $\mathrm{KdV}$ equation is described; a derivation for the special case when the solitary wave and the current are aligned in the same direction is given in the Appendix. Using further simplifications and approximations, a number of analytical expressions are obtained for the variation in the solitary wave amplitude resulting from variable shear in the basic current or from when the basic current is a depth-independent flow which is a simple representation of a geostrophic current, tidal flow or inertial wave.

\section{INTRODUCTION}

Internal solitary waves are a commonly occurring feature in stratified fluids whenever the combination of basic flow stratification and velocity shear permit the formation of a horizontal waveguide. In nature these waves have been observed on the thermoclines of lakes, fjords or in coastal waters (see, for instance, Farmer (1978) or Apel (1980)), and on inversion layers in the atmosphere (see, for instance, Christie et al. (1978) or Clarke et al. (1981)). On the theoretical side it has been established that the appropriate equation to model these waves is either the Korteweg-de Vries equation when the horizontal waveguide has a limited vertical extent (Benney, 1966; 
Maslowe and Redekopp, 1980; Grimshaw, 1981), or an evolution equation of the Benjamin-Ono type for a deep fluid.

Usually the theory has been developed for the case when the basic flow stratification and velocity shear vary only in the vertical direction, with the consequence that the horizontal waveguide is uniform and the model evolution equation has constant coefficients. However, in many natural situations the basic flow is variable with respect to both time and the horizontal coordinates. For example, internal waves in coastal waters propagate over varying bottom topography or through a horizontally sheared current, while in fjords the internal waves often propagate in the presence of temporally and spatially varying tidal currents. Atmospheric internal waves propagate on inversion layers which may erode in time, and through regions of horizontal wind shear. The theoretical basis for the study of these situations are variable coefficient evolution equations of the Korteweg-de Vries family, which have been derived in a general setting by Grimshaw (1981) For the special case of solitary waves on the free surface of a homogeneous fluid (i.e., water waves), the variable coefficient Korteweg-de Vries equation has been derived and discussed by Ostrovsky and Pelinovsky (1970), Kakutani (1971), Johnson (1973), and many others (see the reviews by Miles (1980) or Grimshaw (1986)) for the case when the variability is due to bottom topography, and by Zhou (1985) when the variability is due to a current. Applications for the case of internal solitary waves have been when the variation in the horizontal waveguide is due only to variable bottom topography (see, for instance, Djordevic and Redekopp (1978), or Grimshaw (1983). Here we concentrate on horizontal variability associated with shear in the basic flow, although we shall also consider the effects of variable bottom topography, and some situations where there is also temporal variability. The importance of velocity shear in the basic flow in determining the properties of internal solitary waves has only recently been recognized (Noonan and Smith, 1985; Smyth and Holloway, 1987). For simplicity we shall only consider the case when the waveguide has a limited vertical extent so that the model evolution equation is a variable coefficient Korteweg-de Vries (KdV) equation.

Next we shall describe the variable coefficient $\mathrm{KdV}$ equation derived by Grimshaw (1981) for the general case when the basic stratification and velocity shear vary both spatially and temporally. In the Appendix we describe an alternative derivation when the basic flow is steady and two-dimensional (i.e. the basic horizontal current is uni-directional and varies horizontally only in that direction), and the waves propagate in a direction parallel to the basic flow. For the general case we first introduce non-dimensional coordinates based on a length-scale $h_{1}$ (a typical vertical dimension), a time-scale $N_{1}^{-1}$, where $N_{1}$ is a typical value of the Brunt-Vaisala frequency, 
and pressure-scale $\rho_{1} g h_{1}$ where $\rho_{1}$ is a typical value of the density. These scales combine to form the non-dimensional Boussinesq parameter $\sigma=$ $N_{1}^{2} h_{1} g_{1}^{-1}$, which is a measure of the strength of the basic flow stratification. Relative to these non-dimensional coordinates, the wave field has a lengthscale of $\epsilon^{-1}$, an amplitude-scale of $\epsilon^{2}$, and evolved on time- and space-scales of $\epsilon^{-3}$ where $\epsilon$ is a small parameter. The basic flow is given by a horizontal current $\mathbf{u}_{0}\left(\mathbf{X}^{\prime}, T^{\prime} ; z\right)$ and a density field $\rho_{0}\left(\mathbf{X}^{\prime}, T^{\prime} ; z\right)$, while the Brunt-Vaisala frequency $N$ is given by $\rho_{0 z}=-\sigma \rho N^{2}$. Here $z$ is the vertical coordinate, while

$\mathbf{X}^{\prime}=\epsilon_{\mathrm{x}}^{3}, \quad T^{\prime}=\epsilon^{3} t$

where $\mathbf{x}$ is a horizontal coordinate and $t$ is the time.

The wave field is described by $\epsilon^{2} A(s, \theta) \phi\left(\mathbf{X}^{\prime}, T^{\prime} ; z\right)$, which to leading order is the vertical particle displacement. Here the phase variable is given by

$\theta=\epsilon^{-2} \Theta\left(\mathbf{X}^{\prime}, T^{\prime}\right)$

$\omega=-\Theta_{T^{\prime}}, \quad \kappa=\nabla_{\mathbf{X}^{\prime}} \Theta$

while the vertical modal function is given by

$\left(\rho_{0} \bar{W}^{2} \phi_{z}\right)_{z}+\rho_{0} N^{2} \phi=0$

$\sigma \bar{W}^{2} \phi_{z}-\phi=0$, on $z=\zeta_{0}$

$\phi=0, \quad$ on $z=-h$

$\kappa \bar{W}=\omega-\kappa \cdot \mathbf{u}_{0}$

where $\kappa=|\kappa|$. Here $\zeta_{0}\left(\mathbf{X}^{\prime}, T^{\prime}\right)$ denotes the position of the free surface of the basic flow, and $h\left(\mathbf{X}^{\prime}\right)$ is the fluid depth. Equation (3a) with the boundary conditions ( $3 \mathrm{~b}$ and $\mathrm{c}$ ) define an eigenvalue problem which determines $\omega=\omega\left(\kappa ; X^{\prime}, T^{\prime}\right)$. This is the dispersion relation which, through eqn. (2b), determines the phase $\Theta$. Because of the form of eqn. (3d) it follows that we may put $\omega=\kappa W\left(\kappa^{-1} \kappa ; \mathbf{X}^{\prime}, T^{\prime}\right)$, where the modal equations $(3 \mathrm{a}-\mathrm{c})$ determine $W$, which is the magnitude of the longwave phase speed; $\omega=\kappa W$ is then the dispersion equation for linear longwaves. To solve for the phase $\Theta$ we introduce the rays by the equations

$$
\begin{aligned}
& \frac{\mathrm{d} T^{\prime}}{\mathrm{d} s}=1, \quad \frac{\mathrm{d} \mathbf{X}^{\prime}}{\mathrm{d} s}=\mathbf{V}=\nabla_{\boldsymbol{\kappa}} \omega \\
& \frac{\mathrm{d} \omega}{\mathrm{d} s}=\frac{\partial \omega}{\partial T^{\prime}}, \quad \frac{\mathrm{d} \boldsymbol{\kappa}}{\mathrm{d} s}=-\nabla_{\mathbf{X}^{\prime}} \omega
\end{aligned}
$$

where $s$ is a time-like variable along each ray. Here $\mathbf{V}$ is the group velocity, and we note that it generally differs from the longwave phase speed $\kappa^{-1} \kappa W$ 
because of the possible dependence of $W$ on the wave direction $\boldsymbol{\kappa}^{-1} \boldsymbol{\kappa}$. However, it can be shown that $\omega=\kappa \cdot V$ and hence $\Theta$ is constant on each ray. We let $\xi_{1}^{\prime}=\Theta$ and $\xi_{2}^{\prime}$ be initial coordinates on each ray and then the ray equations have solutions of the form $T^{\prime}=T^{\prime}\left(s, \xi_{1}^{\prime}, \xi_{2}^{\prime}\right)$ and $\mathbf{X}^{\prime}=$ $\mathbf{X}^{\prime}\left(s, \xi_{1}^{\prime}, \xi_{2}^{\prime}\right)$. We let $J$ be the Jacobian of the mapping from $\left(s, \xi_{1}^{\prime}, \xi_{2}^{\prime}\right)$ to $\left(T^{\prime}, \mathbf{X}^{\prime}\right)$.

It is shown by Grimshaw (1981) that the amplitude $A(s, \theta)$ satisfies the variable coefficient $\mathrm{KdV}$ equation

$A_{s}+\kappa \mu A A_{\theta}+\kappa^{3} \lambda A_{\theta \theta \theta}+v A=0$

where the coefficients are given by

$I \mu=3 \int_{-h}^{\zeta_{0}} \rho_{0} \bar{W}^{2} \phi_{z}^{3} \mathrm{~d} z$

$I \lambda=\int_{-h}^{\zeta_{0}} \rho_{0} \bar{W}^{2} \phi^{2} \mathrm{~d} z$

$I=2 \int_{-h}^{\zeta_{0}} \rho_{0} \bar{W} \phi_{z}^{2} \mathrm{~d} z$

The coefficient $v$ is given by

$v=\frac{1}{2} \kappa(I J)^{-1}\left(J I \kappa^{-1}\right)_{s}+\gamma$

where $\gamma$ is due to non-conservative effects, such as friction or forcing, in the basic flow. The general expression for $\gamma$ is quite complicated and will not be displayed here, but we shall quote the results for the special cases discussed in later sections. Note that all the coefficients are functions of $s$, but are independent of $\theta$.

In general, the variable coefficient $\mathrm{KdV}$ equation (5) is not integrable, and we must resort to numerical or approximate solution procedures. Joshi (1987) has shown that those particular choices of coefficients for which eq. (5) is integrable correspond to just those cases for which it can be transformed simply into either a constant coefficient $\mathrm{KdV}$ equation (with $v=0$ ) or into a constant coefficient concentric $\mathrm{KdV}$ equation (with $v=\mathrm{s}^{-1} / 2$ ). The approximate procedures most widely used correspond either to the fission of a solitary wave when there is an abrupt change in the characteristics of the horizontal waveguide, or to the slowly varying solitary wave. Fissioning of internal solitary waves due to topographic variability has been discussed by Djordevic and Redekopp (1978) and Zhou (1987). Here we shall confine our discussion to the slowly varying solitary wave approximation, which assumes that the properties of the waveguide represented by the coefficients in eq. (5) vary slowly with respect to the length-scale of the solitary wave. It has been shown by Grimshaw (1979) that the slowly varying solitary wave solution of eqn. (5) is given by

$A=a \operatorname{sech}^{2} \beta \Psi$ 
where

$\Psi=\theta-\int_{0}^{s} \frac{1}{3} \kappa \mu a \mathrm{~d} s$

and

$\mu a=12 \lambda \kappa^{2} \beta^{2}$

The variation of the amplitude $a$ with $s$ is given by the wave-action flux equation which here takes the form

$\frac{\partial}{\partial s} \int_{-\infty}^{\infty} \frac{I J A^{2}}{\kappa} \mathrm{d} \theta=-2 \gamma \int_{-\infty}^{\infty} \frac{I J A^{2}}{\kappa} \mathrm{d} \theta$

On substituting $(8 \mathrm{a}-\mathrm{c})$ into $(9)$ we find that

$\mu^{-1} \lambda I^{2} J^{2} a^{3}=($ constant $) \exp \left(-4 \int_{0}^{s} \gamma \mathrm{d} s\right)$

Note that when the waveguide is conservative (i.e., $\gamma=0$ ) eqn. (10) gives a local law for the variation of the solitary wave amplitude $a$. Also, it should be noted that the slowly varying solitary wave solution does not, on its own, conserve mass (Grimshaw, 1979; Chang et al. 1979; Knickerbocker and Newell, 1980), and to conserve mass is accompanied by a trailing shelf, or tail. This is a linear longwave where amplitude is much smaller than that of the solitary wave, but which has a much greater spatial extent.

To complete this introductory section we now describe how this general theory reduces under the simplifying hypothesis that the basic flow is steady, two-dimensional and a function of $X^{\prime}$ and $z$ alone. It then follows from eqn. (4b) that $\omega$ and $l$ are constants, where $\kappa=(k, l)$. Without loss of generality we can put $\omega=1$, and the dispersion relation is then $\kappa W=1$, where $\kappa=\left(k^{2}+l^{2}\right)^{1 / 2}$. This determines $k=k\left(X^{\prime}\right)$, since now $W=$ $W\left(\kappa^{-1} k ; X^{\prime}\right)$. Note that the modal equations ( $3 \mathrm{a}$ and b) contain only $\bar{W}=W-\kappa^{-1} k u_{0}$, and that the group velocity $\mathbf{V}=\left(V_{1}, V_{2}\right)$ where $V_{1}=$ $\partial / \partial k(\kappa W), V_{2}=\partial / \partial l(\kappa W)$. Clearly we can regard $k, W, V_{1}$ and $V_{2}$ as functions of $X^{\prime}$ alone, with the constant $l$ as a parameter. Then the ray equations have the solution

$T^{\prime}=s-\xi_{1}^{\prime}+l \xi_{2}^{\prime}, \quad s=\int_{0}^{X^{\prime}} V_{1}^{-1} \mathrm{~d} X^{\prime}, \quad Y^{\prime}=\xi_{2}^{\prime}+\int_{0}^{X^{\prime}} V_{2} V_{1}^{-1} \mathrm{~d} X^{\prime}$

$\Theta=\xi_{1}^{\prime}, \quad \omega=1, \quad \kappa W=1$

Note that it may be shown that

$\Theta=-T^{\prime}+\int_{0}^{X^{\prime}} k \mathrm{~d} X^{\prime}+l Y^{\prime}$ 
and we recall from eqn. (2a) that $\theta=\epsilon^{-2} \Theta$. The Jacobian $J=V_{1}$. When these results are substituted into eqn. (5) we obtain

$A_{s}+W^{-1} \mu A A_{\theta}+W^{-3} \lambda A_{\theta \theta \theta}+\nu A=0$

where

$\nu=\frac{1}{2}\left(W V_{1} I\right)^{-1}\left(W V_{1} I\right)_{s}+\gamma$

For the special case when the solitary waves also propagate in the $X^{\prime}$ direction, we may put $l=0$. Then it follows that $W=W\left(X^{\prime}\right), k=W^{-1}$, $V_{1}=W$ and $V_{2}=0$. Since the derivation of the variable coefficient $\mathrm{KdV}$ equation in the general case (Grimshaw, 1981) is rather abstract and lengthy, we present in the Appendix a brief account of a more direct derivation for this special case.

2. APPLICATIONS IN THE ONE-DIMENSIONAL CASE FOR A STEADY BASIC FLOW

When the basic flow is independent of $Y^{\prime}$ and $T^{\prime}$, and is directed in the $X^{\prime}$ direction (i.e., is steady, two-dimensional and a function of $X^{\prime}$ and $z$ alone) then the reduction of the general theory was described at the end of the last section, and the equation to be considered is the variable coefficient $\mathrm{KdV}$ equation (13a). Note that in the title of this section we have called this the one-dimensional case because the basic flow has only one horizontal component which points in the $X^{\prime}$ direction, although, of course, the total flow is two-dimensional, and the solitary waves may propagate in a direction different to the $X^{\prime}$ direction (if $l \neq 0$ ).

For specific applications we must now specify the basic horizontal velocity field $\left(u_{0}\left(X^{\prime}, z\right)\right.$. Once this has been done, the general strategy is to solve the modal equations ( $3 \mathrm{a}-\mathrm{d})$ and hence find $W$, calculate the coefficients $\mu, \lambda$ and $\nu$ from eqns. (6a-c) and (7), and then determine the variation of the solitary wave amplitude from eqn. (10). For any given $u_{0}\left(X^{\prime}, z\right)$ this procedure can, of course, be carried out numerically. However, our aim here is to obtain analytical expressions for the solitary wave amplitude, and, as we shall see, the greatest difficulty with this strategy is obtaining sufficiently simple expressions for the basic flow so that the modal equations can be solved analytically.

The basic flow now satisfies the eqns. (A.1a-d). The solution of (A.1c and d) is given by

$$
\begin{aligned}
& u_{0}=\Psi_{z}, \quad w_{0}=-\Psi_{X^{\prime}} \\
& \rho_{0}=\rho_{0}(\Psi)
\end{aligned}
$$


The functional form of $\rho_{0}(\Psi)$ can generally be determined by evaluating $\rho_{0}$ and $\Psi$ far upstream, as $X^{\prime} \rightarrow-\infty$. Eliminating $p_{0}$ from eqn. (A.1a and b) we find that

$\rho_{0} J(\eta, \Psi)=F_{0 z}-\epsilon^{6} G_{0 X^{\prime}}$

where

$\eta=\Psi_{z z}+\epsilon^{6} \Psi_{X^{\prime} X^{\prime}}+\rho_{0}^{-1} \frac{\mathrm{d} \rho_{0}}{\mathrm{~d} \Psi}\left(\sigma^{-1} z+\frac{1}{2} u_{0}^{2}+\frac{1}{2} \epsilon^{6} w_{0}^{2}\right)$

$J(A, B)=A_{X^{\prime}} B_{z}-A_{z} B_{X}$

In the absence of the external forces $F_{0}$ and $G_{0}$ eqn. (15a) has the solution $\eta=\eta(\Psi)$, which can be recognized as Long's equation for the steady two-dimensional flow of a stratified fluid (Long, 1953). The boundary conditions (A.2a and c) become

$\Psi=0, \quad$ on $z=\zeta_{0}\left(X^{\prime}\right)$

$\Psi=-Q, \quad$ on $z=-h\left(X^{\prime}\right)$

where $Q$ is a constant describing the total mass flux of the basic flow. The boundary condition (A.2b) becomes

$\rho_{0} \zeta_{0 X^{\prime}}+\sigma \rho_{0} \frac{\mathrm{d}}{\mathrm{d} X^{\prime}}\left[\left(\frac{1}{2} u_{0}^{2}+\frac{1}{2} \epsilon^{6} w_{0}^{2}\right)_{z=\zeta_{0}}\right]=\sigma\left(F_{0}+\epsilon^{6} G_{0} \zeta_{0 X^{\prime}}\right), \quad$ on $z=\zeta_{0}\left(X^{\prime}\right)$

The basic flow is thus described by eqn. (15a), with boundary conditions $(16 \mathrm{a}$ and $\mathrm{b})$ and (17). The $O\left(\epsilon^{6}\right)$ terms may be omitted. Even so, however, these equations are non-linear, and further simplifications and approximations are needed. We shall exploit the Boussinesq limit $\sigma \rightarrow 0$, as well as other simplifications. For applications to internal solitary waves in the atmosphere or ocean, $\sigma$ is typically in the range $10^{-2}$ to $10^{-3}$.

\subsection{Flow over topography}

We shall suppose that $h\left(X^{\prime}\right) \rightarrow h_{0}$ as $X^{\prime} \rightarrow-\infty$, and that upstream

$\left.\begin{array}{l}u_{0} \rightarrow U_{0}+\alpha z \\ \rho_{0} \rightarrow \exp \left(-\sigma N_{0}^{2} z\right)\end{array}\right\}$ as $X^{\prime} \rightarrow-\infty$

Where $U, \alpha$ and $N_{0}$ are constants. Hence

$\Psi \rightarrow U_{0} z+\frac{1}{2} \alpha z^{2}, \quad$ as $X^{\prime} \rightarrow-\infty$

and elimination of $z$ between (18b) and (19) defines $\rho_{0}(\Psi)$. For this case we 
shall suppose that the $X^{\prime}$ variation in the basic flow is solely due to the topography, and hence we can put the external forces $F_{0}$ and $G_{0}$ equal to zero. Equation (18b) can then be integrated to give

$\eta=\eta(\Psi)$

where the functional form of $\eta(\Psi)$ is determined by considering the limit $X^{\prime} \rightarrow-\infty$. In order to obtain a tractable equation we exploit the limits $\sigma \rightarrow 0$ and $\alpha h_{0} / U_{0} \rightarrow 0$. The assumption that $\alpha h_{0} / U_{0}$ is small is not a major restriction on applications. For instance $\alpha=10^{-2}$ allows for a velocity difference of $1 \mathrm{~m} \mathrm{~s}^{-1}$ over a $100 \mathrm{~m}$ depth range. It may then be shown that eqn. (20) becomes

$\Psi_{z z}+\beta^{2}\left(\Psi-U_{0 z}\right)=\alpha\left[1-\beta^{2}\left(\frac{z \Psi}{U_{0}}-\frac{3 \Psi^{2}}{2 U_{0}^{2}}\right)\right]+\frac{1}{2} \alpha \beta^{2}\left(U_{0} \Psi_{z}^{2}-U_{0}^{3}\right)+\ldots$

where

$\beta=N_{0} U_{0}^{-1}$

and the omitted terms in (21a) are higher order terms in $\sigma$ and $\alpha$. The boundary conditions (16a and b) become

$$
\begin{aligned}
& \Psi+\frac{1}{2} \sigma \Psi_{z}\left(U^{2}-\Psi_{z}^{2}\right)+--=0, \quad \text { on } z=0 \\
& \Psi=-U_{0} h_{0}+\frac{1}{2} \alpha h_{0}^{2}, \quad \text { on } z=-h\left(X^{\prime}\right)
\end{aligned}
$$

To leading order, the solution of (21a) and (22a and b) is

$$
\Psi=U_{0} z+U_{0}\left(h_{0}-h\right) \frac{\sin \beta z}{\sin \beta h}+0\left(\sigma, \alpha h_{0} / U_{0}\right)
$$

However, although the $O(\sigma, \alpha)$ terms are readily calculated the corresponding expressions are still too complicated to allow us to obtain analytical solutions of the modal equations $(3 a-c)$. Hence we make the further approximation $\beta h_{0} \rightarrow 0$, which can be regarded as a large internal Froude number approximation, and restricts our results to relatively strong currents, or winds. We find that

$$
\begin{aligned}
\Psi= & \frac{U_{0} h_{0} z}{h}+\frac{\beta^{2} U_{0}\left(h_{0}-h\right)\left(h^{2}-z^{2}\right) z}{6 h}+\frac{\sigma U_{0}^{3} h_{0}\left(h_{0}^{2}-h^{2}\right) z}{2 h^{4}} \\
& +\frac{1}{2} \alpha\left[z^{2}-\left(\frac{h_{0}^{2}-h^{2}}{h}\right) z\right]+\cdots
\end{aligned}
$$


Finally $u_{0}$ and $\rho_{0}$ are found from eqn. (14a and b), and are given by

$$
\begin{aligned}
u_{0}= & \frac{U_{0} h_{0}}{h}+\frac{\beta^{2} U_{0}\left(h_{0}-h\right)\left(h^{2}-3 z^{2}\right)}{6 h}+\frac{\sigma U_{0}^{3} h_{0}\left(h^{2}-h^{2}\right)}{2 h^{4}} \\
& +\alpha\left[z-\frac{\left(h_{0}^{2}-h^{2}\right)}{2 h}\right]+\cdots \\
\frac{N^{2}}{N_{0}^{2}}= & \frac{h_{0}}{h}+\frac{\beta^{2}\left(h_{0}-h\right)\left(h^{2}-3 z^{2}\right)}{\subset h}+\frac{\sigma U_{0}^{2} h_{0}\left(h_{0}^{2}-h^{2}\right)}{U h^{4}} \\
& -\frac{\alpha\left(h_{0}^{2}-h^{2}\right)\left(1+\frac{2 z}{h}\right)}{2 h U_{0}}+\cdots
\end{aligned}
$$

These expressions hold in the joint approximations of large internal Froude number $\left(\beta h_{0} \ll 1\right)$, weak shear $\left(\alpha h_{0} \ll U_{0}\right)$, and the Boussinesq limit $(\sigma \ll$ 1.)

We turn next to the solution of the modal equations ( $3 a-d)$. To leading order the modal function and $W$ are given by

$$
\begin{aligned}
& \phi=\sin (s \pi z / h)+0\left(\sigma, \alpha h_{0} / U_{0}, \beta^{2} h_{0}^{2}\right) \\
& W=\frac{k U_{0} h_{o}}{\kappa h}+\frac{N_{0}\left(h h_{0}\right)^{\frac{1}{2}}}{s \pi}+0\left(\sigma, \alpha h_{0} / U_{0}, \beta^{2} h_{0}^{2}\right)
\end{aligned}
$$

where $s=1,2,3, \cdots$, and we recall $\kappa=(k, l), \kappa=\left(k^{2}+l^{2}\right)^{\frac{1}{2}}$ and is a constant. Since $\kappa W=1$, eqn. (26b) determines the variation of $k$ with $h\left(X^{\prime}\right)$. Note that the second term of eqn. $(26 \mathrm{~b})$ is $0\left(\beta h_{0}\right)$ relative to the first, and hence $k$ varies approximately in proportion to $h$. The group velocity is given by

$$
\begin{aligned}
& V_{1}=\frac{U_{0} h_{0}}{h}+\frac{k N_{0}\left(h h_{0}\right)^{\frac{1}{2}}}{\kappa s \pi}+0\left(\frac{\sigma, \alpha h_{0}}{U_{0}, \beta^{2} h_{0}^{2}}\right) \\
& V_{2}=\frac{l N_{0}\left(h h_{0}\right)^{1 / 2}}{\kappa s \pi}+0\left(\sigma, \alpha h_{0} / U_{0}, \beta^{2} h_{0}^{2}\right)
\end{aligned}
$$

Here, in the expression for $V_{1}$ the first term dominates, and so $V_{1}$ varies approximately in inverse proportion to $h$. It can also be shown that $V_{2} / V_{1}$ is an increasing function of $h$. The solution of the ray equations is given by eqns. (11a and b) and (12), and the Jacobian $J=V_{1}$. Although the second term in eqn. ( $26 \mathrm{~b})$ is $O\left(\beta h_{0}\right)$ relative to the first, it has been retained as it is 
the leading order term in $\bar{W}$, where here $\bar{W}=W-\kappa^{-1} k u_{0}$. Next we calculate the coefficients $\mu, \lambda$ and $I$ from eqn. $(6 \mathrm{a}-\mathrm{c})$. We find that

$I=N_{0} s \pi\left(h_{0} / h\right)^{\frac{1}{2}}+0\left(\sigma, \alpha h_{0} / U_{0}, \beta^{2} h_{0}^{2}\right)$

$\lambda=\frac{1}{2} N_{0}(h / s \pi)^{3}\left(h_{0} / h\right)^{\frac{1}{2}}+0\left(\sigma, \alpha h_{0} / U_{0}, \beta^{2} h_{0}^{2}\right)$

However $\mu$ (eqn. (6a)) is itself $0\left(\sigma, \alpha h_{0} / U_{0}, \beta h_{0}\right)$ and to calculate it directly from eqn (6a) requires evaluation of the $0\left(\sigma, \alpha h_{0} / U_{0}, \beta h_{0}\right)$ terms in $\phi$ (eqn. (26a)) and $W$ (eqn. 26(b)). Alternatively, we integrate eqn. (6a) by parts to obtain the equivalent expression

$$
\begin{aligned}
I \mu= & 3 \int_{-h}^{\zeta_{0}}\left(\rho_{0} \bar{W}^{2}\right)_{z} \phi \phi_{z}^{2} \mathrm{~d} z-2 \int_{-h}^{\zeta_{0}}\left(\rho_{0} N^{2}\right)_{z} \phi^{3} \mathrm{~d} z+3 \sigma\left(\rho_{0} \bar{W}^{4} \phi_{z}^{3}\right)_{z=\zeta_{0}} \\
& +2 \sigma^{3}\left(\rho_{0} N^{2} \bar{W}^{6} \phi_{z}^{3}\right)_{z=\zeta_{0}}
\end{aligned}
$$

From this expression we can calculate $\mu$ directly from eqn. (26a and b) and find that, to leading order

$$
\begin{aligned}
\mu= & \sigma\left[3-\frac{1}{3}\left(1-(-1)^{s}\right)\right] N_{0}^{3}\left(\frac{h_{0}}{s \pi}\right)^{2}\left(h h_{0}\right)^{-\frac{1}{2}} \\
& +\alpha\left[1-(-1)^{s}\right]\left[\frac{2 k}{\kappa} \frac{1}{s \pi}-\frac{4 N_{0}}{3 U_{0}} \frac{1}{(s \pi)^{2}}\left(h_{0}^{2}-h^{2}\right)\left(h h_{0}\right)^{-\frac{1}{2}}\right] \\
& -\beta^{2}(-1)^{s}\left[\frac{h_{0}-h}{h_{0}}\right]\left[\frac{2 k}{\kappa} \frac{U_{0} h_{0}}{s \pi}+\frac{4 N_{0}}{3}\left(\frac{h}{s \pi}\right)^{2}\left(\frac{h_{0}}{h}\right)^{\frac{1}{2}}\right]+\cdots
\end{aligned}
$$

In the coefficients of both $\alpha$ and $\beta$ in this expression the second term is $O\left(\beta h_{0}\right)$ relative to the first term, and hence can be neglected. Also the ' $\sigma$ ' term in eqn. (30) is $0\left(\beta h_{0}\right)$ relative to the ' $\beta^{2} h_{0}^{2}$ ' term, and can also be neglected.

Finally, the variation of the solitary wave amplitude is determined from eqn. (10) with the friction coefficient $\gamma=0$. Thus

$a^{3}=($ constant $) \mu / \lambda I^{2} V_{1}^{2}$

where $V_{1}, I, \lambda$ and $\mu$ are given by eqns (27a), (28a and b) and (30), respectively. Utilizing the further approximations outlined above we find that, to leading order

$$
\begin{aligned}
a^{3}= & (\text { constant }) \frac{k}{\kappa}\left(\frac{h}{h_{0}}\right)^{\frac{1}{2}}\left\{\alpha\left[1-(-1)^{s}\right]-\beta^{2}(-1)^{s} U_{0}\left(h_{0}-h\right)\right\} \\
& +\sigma(\text { constant })
\end{aligned}
$$




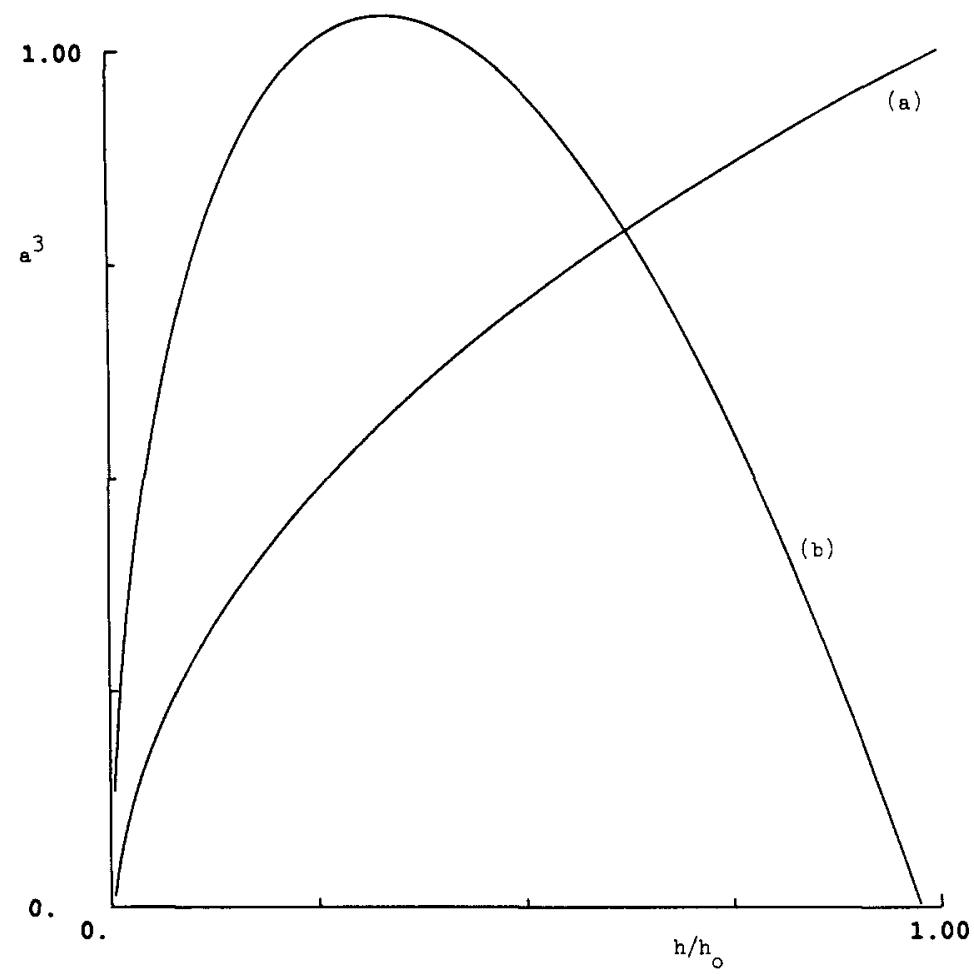

Fig. 1. A plot of the solitary wave amplitude $a$ as a function of the depth $h$ for case (a) of section 2, when $l=0, \alpha h_{0} / U_{0}=\beta h_{0}=0.1$ and $\sigma N_{0}^{2} h_{0}=0.01$. The curve (a) is for the mode $s=1$, and (b) is for $s=2$. Note that the two curves have been normalized differently.

where we recall that, to leading order, $k$ varies approximately in proportion to $h$. Thus, for instance, if the solitary wave propagates in the direction in which the depth $h$ is decreasing, then $k$ decreases and so the wave crest tends to align parallel to the current (which is in the $X^{\prime}$ direction). On the other hand, $V_{2} / V_{1}$ decreases as $h$ decreases and so the ray direction tends to refract towards the basic current. If we assume that the mode number $s$ is an odd integer, and that the ' $\alpha h_{0} / U_{0}$ ' term in eqn. (32) dominates over the ' $\beta^{2} h_{0}^{2}$ ' term, then the solitary wave amplitude decreases. However, if the mode number $s$ is an even integer, or the ' $\beta^{2} h_{0}^{2}$ ' term in eqn. (32) dominates, then the solitary wave amplitude at first increases, but then decreases. We also note that the ' $\sigma$ ' term in eqn. (32) produces no change in the amplitude as the depth $h$ varies. In Fig. 1 we show a plot of the solitary wave amplitude ' $a$ ' as a function of $h$ for the special case $l=0$ for some representative values of $\alpha h_{0} / u_{0}, \beta h_{0}$ and $\sigma N_{0}^{2} h_{0}$, and for the modes $s=1$ and $s=2$. The cases when $l \neq 0$ are similar.

We shall conclude this subsection by contrasting these results with the corresponding results when $u_{0} \equiv 0$ (see, for instance, Grimshaw (1983)). In 
this case $\rho_{0}=\exp \left(-\sigma N_{0}^{2} Z\right)$ for all $X^{\prime}$, and the solution of the modal equations $(3 a-d)$ is

$$
\begin{aligned}
& \phi=\sin (s \pi z / h)+0(\sigma) \\
& W=N_{0} h / s \pi+0(\sigma)
\end{aligned}
$$

Since $\kappa W=1, \kappa$ now varies inversely with $h$, and as the solitary wave propagates in the direction in which the depth $h$ is decreasing, then $\kappa$ increases and the wave crests tend to align normal to the $X^{\prime}$ direction. Also $V_{2} / V_{1}=l / k$ and hence decreases as $h$ decreases, and so the ray direction refracts towards the $X^{\prime}$ direction. Here $V_{1}=k W / \kappa$. The coefficients $\mu, \lambda$ and $I$ are given by

$$
\begin{aligned}
& \mu=\sigma\left\{3-\frac{1}{3}\left[1-(-1)^{s}\right]\right\} N_{0}^{3} h /(s \pi)^{2}+0\left(\sigma^{2}\right) \\
& \lambda=N_{0} h^{3} / 2(s \pi)^{3}+0(\sigma) \\
& I=N_{0} s \pi+0(\sigma)
\end{aligned}
$$

The solitary wave amplitude is again given by eqn. (31), which reduces to

$a^{3}=($ constant $) \kappa^{2} / k^{2} h^{4}+0(\sigma)$

Thus as the depth decreases, the solitary wave amplitude now increases. These results are in direct contrast to those given above when a basic current is present (i.e. $u_{0} \neq 0$ ), and emphasize the importance of considering the effect of currents when evaluating the properties of internal solitary waves.

\subsection{Flow with variable shear}

We shall now suppose that the depth $h$ is a constant, and that the basic flow is given by

$$
\begin{aligned}
& \Psi=U_{0} z+\alpha\left(X^{\prime}\right)\left(z^{2}+h z\right) \\
& u_{0}=U_{0}+\alpha\left(X^{\prime}\right)(2 z+h)
\end{aligned}
$$

Note that $\Psi$ satisfies the boundary conditions (16a and b), where we are assuming that the free surface is $\zeta_{0}=0$. Here $\alpha\left(X^{\prime}\right)$ is an arbitrary shear, although we shall suppose for simplicity that $\left|\alpha h_{0}\right| \ll U_{0}$. The density is given by

$\rho_{0}=\exp \left(-\sigma N_{0}^{2} \Psi / U_{0}\right)$

so that the Brunt-Vaisala frequency $N$ is constant to leading order. This flow must be maintained by an external force $F_{0}$, which is given by (see A. 1a))

$F_{0}=\rho_{0} U_{0} \boldsymbol{\alpha}_{X^{\prime}}\left\{2 z+h+\beta^{2}\left(\frac{1}{3} z^{3}+\frac{1}{2} h z^{2}\right)\right\}+---$ 
where the omitted terms are $0\left(\sigma^{2}, \sigma \alpha h / U_{0}, \alpha^{2} h^{2} / U_{0}^{2}\right)$ and $\beta$ is again given by eqn. (21b).

The solution of the modal equations $(3 a-d)$ is

$$
\begin{aligned}
& \phi=\sin (s \pi z / h)+0\left(\sigma, \alpha h / U_{0}\right) \\
& W=\frac{k U_{0}}{\kappa}+\frac{N_{0} h}{s \pi}+0\left(\sigma, \alpha h / U_{0}\right)
\end{aligned}
$$

where again $s=1,2,3 \ldots$. Since $\kappa W=1$ it follows that here $k$, and hence $\kappa$, is a constant to leading order. Similarly $V_{1}$ is a constant to leading order, while $V_{2} / V_{1}$ is proportional to $h$ to leading order. Hence the wave crests have a constant alignment to the current direction, while the ray direction refracts towards the current direction. The coefficients $\mu, I$ and $\lambda$ are now given by

$$
\begin{aligned}
\mu= & \alpha\left[1-(-1)^{s}\right]\left[\frac{4 k}{\kappa} \frac{1}{s \pi}+\frac{8}{3} \frac{N_{0} h}{U_{0}(s \pi)^{2}}\right]+\sigma\left\{3-\frac{1}{3}\left[1-(-1)^{s}\right]\right\} \frac{N_{0}^{3} h}{(s \pi)^{2}} \\
& +- \\
\lambda= & N_{0} h^{3} / 2(s \pi)^{3}+0\left(\sigma, \alpha h_{0} / U_{0}\right) \\
I= & N_{0} s \pi+0\left(\sigma, \alpha h_{0} / U_{0}\right)
\end{aligned}
$$

The coefficient $\gamma$ is calculated from eqn. (A.15) and we find that

$\gamma=0\left(\sigma^{2}, \sigma \alpha h / U_{0}, \alpha^{2} h^{2} / U_{0}^{2}\right)$

Hence, the variation of the solitary wave amplitude is again determined by eqn. (31), which reduces to

$a^{3}=($ constant $) \alpha\left[1-(-1)^{s}\right]+\sigma($ constant $)$

Consider, for instance, the situation when $\alpha \rightarrow 0$ upstream, as $X^{\prime} \rightarrow-\infty$. The solitary wave, propagating in the positive $X^{\prime}$ direction, is then advancing into a region of increasing shear as $|\alpha|$ increases. When the mode number $s$ is an even integer, the solitary wave amplitude is unaffected by the shear to this order. However, when the mode number $s$ is an odd integer, the solitary wave amplitude increases if $\alpha$ is positive, but decreases if $\alpha$ is negative.

\section{APPLICATIONS IN THE TWO-DIMENSIONAL CASE}

Our aim here is to model situations in which internal solitary waves are propagating through geostrophic currents, tidal flows or inertial waves. Thus we shall assume that the basic flow is depth-independent, with two horizon- 
tal components, and is driven by Coriolis forces. It is given by $\mathbf{u}_{0}=$ $\left(\mathrm{u}_{0}\left(X^{\prime}\left(Y^{\prime}, T^{\prime}\right), v_{0}\left(X^{\prime}, Y^{\prime}, T^{\prime}\right), 0\right)\right.$ and satisfies the approximate equations (see, for instance, Gill (1982)).

$$
\begin{aligned}
& u_{0 T^{\prime}}+\sigma^{-1} \zeta_{0 X^{\prime}}=f v_{0^{\prime}} \\
& v_{0 T^{\prime}}+\sigma^{-1} \zeta_{0 Y^{\prime}}=-f u_{0^{\prime}} \\
& \zeta_{0 T^{\prime}}+h\left(u_{0 X^{\prime}}+v_{0 Y^{\prime}}\right)=0
\end{aligned}
$$

Here we have assumed that the depth $h$ is a constant and $f$ is the Coriolis parameter. In the general formulation of Grimshaw (1981), the Coriolos terms are regarded as body forces in the equations of motion, and it can be shown that their contribution to $\gamma$ in eqn. (7) is $0\left(f^{2}\right)$, where $f=f_{1} N_{1}^{-1}, f_{1}$ being the dimensional value of the Coriolos parameter and $N_{1}$ being a typical value of the Brunt-Vaisala frequency. Since this is a very small term in practice we shall suppose that $\gamma$ is zero, although of course, the Coriolis terms are important in eqn. (43a and $b$ ) in determining the basic flow. Also, since $\zeta_{0}$ is $O(\sigma f)$, it is also small, and can be taken as zero in expressions such as $(6 \mathrm{a}-\mathrm{c})$. Further, we note that the density $\rho_{0}$ is independent of $X^{\prime}$, $Y^{\prime}, T^{\prime}$ to leading order, and we assume that $\rho_{0}=\rho_{0}(z)$. It follows that the modal equations reduce to

$$
\begin{aligned}
& \left(\rho_{0} \phi_{z}\right)_{z}+\rho_{0} \frac{N^{2}}{c^{2}} \phi=0 \\
& \sigma c^{2} \phi_{z}-\phi=0, \quad z=0 \\
& \phi=0, \quad z=-h \\
& W=\frac{\boldsymbol{\kappa} \cdot \mathbf{u}_{0}}{\kappa}+c
\end{aligned}
$$

Here $c=\bar{W}$ is a constant, and the modal functions $\phi=\phi(z)$ are independent of $X^{\prime}, Y^{\prime}, T^{\prime}$. It follows that the coefficients $\mu, \lambda$ and $I$ (eqn. (6a-c)) are also constants, and the only quantities which vary in the $\mathrm{KdV}$ equation (5) are $\kappa$ and $J$. Further the expression (10) for the solitary wave amplitude reduces to

$a^{3}=($ constant $) J^{-2}$

Finally, to determine $\kappa$ and $J$ we must consider the ray equations (4a and b). These become

$$
\begin{aligned}
& \frac{\mathrm{d} T^{\prime}}{\mathrm{d} s}=1, \quad \frac{\mathrm{d} X^{\prime}}{\mathrm{d} s}=\frac{k c}{\kappa}+u_{0}, \quad \frac{\mathrm{d} Y^{\prime}}{\mathrm{d} s}=\frac{l c}{\kappa}+v_{0} \\
& \frac{\mathrm{d} \omega}{\mathrm{d} s}=\kappa \cdot \mathbf{u}_{0 T^{\prime}}, \quad \frac{\mathrm{d} k}{\mathrm{~d} s}=-\kappa \cdot \mathbf{u}_{0 X^{\prime}}, \quad \frac{\mathrm{d} l}{\mathrm{~d} s}=-\kappa \cdot \mathbf{u}_{0 Y^{\prime}}
\end{aligned}
$$

To make further progress we now consider a number of special cases. 


\subsection{Geostrophic current}

We consider the special case when $u \equiv 0$, and $v_{0}=v_{0}\left(X^{\prime}\right)$, and so $\zeta_{0 X^{\prime}}=\sigma f v_{0}$. The ray equations $(46 \mathrm{a}$ and $\mathrm{b})$ reduce to

$$
\begin{aligned}
& \frac{\mathrm{d} T^{\prime}}{\mathrm{d} s}=1, \quad \frac{\mathrm{d} X^{\prime}}{\mathrm{d} s}=\frac{k c}{\kappa}, \quad \frac{\mathrm{d} Y^{\prime}}{\mathrm{d} s}=\frac{l c}{\kappa}+v_{0}, \\
& \frac{\mathrm{d} \omega}{\mathrm{d} s}=0, \quad \frac{\mathrm{d} k}{\mathrm{~d} s}=-v_{0 X^{\prime}}, \quad \frac{\mathrm{d} l}{\mathrm{~d} s}=0
\end{aligned}
$$

It follows that $\omega$ and $l$ are constants, and without loss of generality we put $\omega=1$. The dispersion relation is then $\kappa W=1$, where $\kappa=\left(k^{2}+l^{2}\right)^{\frac{1}{2}}$ and here $W=\frac{l v_{0}}{\kappa}+c$

This equation thus determines $k=k\left(X^{\prime}\right)$. The ray equations then have the solution

$$
\begin{aligned}
& T^{\prime}=s-\xi_{1}^{\prime}+l \xi_{2}^{\prime}, \quad s=\frac{1}{c} \int_{0}^{X^{\prime}} \frac{\kappa \mathrm{d} X^{\prime}}{k} \\
& Y^{\prime}=\xi_{2}^{\prime}+\int_{0}^{X^{\prime}}\left(\frac{l}{k}+\frac{\kappa v_{0}}{k c}\right) \mathrm{d} x^{\prime} \\
& \Theta=\xi_{1}^{\prime}, \quad \omega=1, \quad \kappa W=1
\end{aligned}
$$

Note that it may be shown that

$\Theta=-T^{\prime}+\int_{0}^{X^{\prime}} k \mathrm{~d} X^{\prime}+l Y^{\prime}$

The Jacobian $J=k c / \kappa$ and $V_{2} / V_{1}=\left(\kappa V_{0}+l c\right) / k c$. The variation of the solitary wave amplitude is now determined from eqn. (45) and the relation $\kappa W=1$. Assuming that $k c>0$ so that the solitary wave propagates in the positive $X^{\prime}$ direction we find that as $v_{0}$ increases (decreases), the amplitude also increases (decreases), $k$ decreases (increases) so that wave crests tend to align parallel (normal) to the $X^{\prime}$ direction, and $V_{2} / V_{1}$ increases (decreases) so that the rays refract away from (towards) the $X^{\prime}$ direction.

\subsection{Tidal flow.}

We consider a steady wave-like solution of eqn. (43a-c), for which the solution is

$u_{0}=n \sin \left(m X^{\prime}+n Y^{\prime}\right)$

$v_{0}=-m \sin \left(m X^{\prime}+n Y^{\prime}\right)$ 
very simple model of a tidal flow in which the time variation of the tidal current is neglected on the grounds that the internal solitary wave has a propagation time generally somewhat less than a tidal period. The ray equations ( $46 a$ and $b$ ) reduce to

$$
\begin{aligned}
& \frac{\mathrm{d} T^{\prime}}{\mathrm{d} s}=1, \quad \frac{\mathrm{d} X^{\prime}}{\mathrm{d} s}=\frac{k c}{\kappa}+n \sin \left(m X^{\prime}+n Y^{\prime}\right) \\
& \frac{\mathrm{d} Y^{\prime}}{\mathrm{d} s}=\frac{l c}{\kappa}-m \sin \left(m X^{\prime}+n Y^{\prime}\right) \\
& \frac{\mathrm{d} \omega}{\mathrm{d} s}=0, \quad \frac{\mathrm{d} k}{\mathrm{~d} s}=m(m l-n k) \cos \left(m X^{\prime}+n Y^{\prime}\right) \\
& \frac{\mathrm{d} l}{\mathrm{~d} s}=n(m l-n k) \cos \left(m X^{\prime}+n Y^{\prime}\right)
\end{aligned}
$$

From eqn. (52b) we see that $\omega$ and $(m l-n k)$ are constants. Without loss of generality we put $\omega=1$, and so

$\theta_{0} \sin \left(m X^{\prime}+n Y^{\prime}\right)+\kappa c=1$

$n k-m l=\theta_{0}$

where $\theta_{0}$ is a constant. These two equations together determine $k, l$ as functions of $\left(m X^{\prime}+n Y^{\prime}\right)$, and then eqns. (52a and b) can be solved. Indeed, eqns ( $52 \mathrm{a}$ and b) can be transformed into a form similar to eqn. (47a) by using $\left(m X^{\prime}+n Y^{\prime}\right)$ and $\left(-n X^{\prime}+m Y^{\prime}\right)$ as new variables in place of $X^{\prime}, Y^{\prime}$. We shall omit details, as the conclusion is that

$J=\frac{(m k+n l) c}{\left(m^{2}+n^{2}\right)^{\frac{1}{2}} k}$

The variation of the solitary wave amplitude is now determined from eqn. (45) and the relations (53a and b). Assuming that $(m k+n l) c>0$ so that the solitary wave propagates in the direction in which $\left(m X^{\prime}+n Y^{\prime}\right)$ increases, we find that as $\theta_{0} \sin \left(m X^{\prime}+n Y^{\prime}\right)$ increases (decreases), the amplitude also increases (decreases), and $(m k+n l)$ decreases (increases) so that the wave crests tend to align parallel (normal) to the $\left(m X^{\prime}+n Y^{\prime}\right)$ direction, while the rays refract away from (towards) the $\left(m X^{\prime}+n Y^{\prime}\right)$ direction. The special case $\theta_{0}=0$ is interesting as then the solitary wave is always propagating at right angles to the current, where $k$ and $l$ are constants, so that $J$ is constant, and the solitary wave amplitude remains unchanged. 


\subsection{Inertial current}

In this case the solution of eqn. $(43 a-c)$ is $\zeta_{0} \equiv 0$ and

$u_{0}=U_{0} \sin f T^{\prime}$

$v_{0}=U_{0} \cos f T^{\prime}$

The ray equations ( $46 \mathrm{a}$ and $\mathrm{b})$ become

$\frac{\mathrm{d} T^{\prime}}{\mathrm{d} s}=1, \quad \frac{\mathrm{d} X^{\prime}}{\mathrm{d} s}=\frac{k c}{\kappa}+u_{0} \sin f T^{\prime}, \quad \frac{\mathrm{d} Y^{\prime}}{\mathrm{d} s}=\frac{l c}{\kappa}+U_{0} \cos f T^{\prime}$,

$\frac{\mathrm{d} \omega}{\mathrm{d} s}=f U_{0}\left(k \cos f T^{\prime}-l \sin f T^{\prime}\right), \quad \frac{\mathrm{d} k}{\mathrm{~d} s}=0, \quad \frac{\mathrm{d} l}{\mathrm{~d} s}=0$

From eqn. (56b) we see that $k$ and $l$ are constants and then $\omega$ is given by

$\omega=\kappa W=\kappa c+U_{0}\left(k \sin f T^{\prime}+l \cos f T^{\prime}\right)$

The solution of eqn. (56a and $b$ ) is then

$T^{\prime}=s$

$X^{\prime}=\frac{k \xi_{1}^{\prime}}{\kappa}-\frac{l \xi_{2}^{\prime}}{\kappa}+\frac{k c}{\kappa} T^{\prime}-\frac{U_{0}}{f} \cos f T^{\prime}$

$Y^{\prime}=\frac{l \xi_{1}^{\prime}}{\kappa}+\frac{k \xi_{2}^{\prime}}{\kappa}+\frac{l c}{\kappa} T^{\prime}+\frac{U_{0}}{f} \sin f T^{\prime}$

and the phase is given by

$\Theta=-\kappa c T^{\prime}+\frac{U_{0}}{f}\left(k \cos f T^{\prime}-l \sin f T^{\prime}\right)+k X^{\prime}+l Y^{\prime}$

The Jacobian $J=\kappa$, and is constant. Hence the solitary wave amplitude does not vary as it propagates in an inertial current.

\section{SUMMARY}

Our main aim in this paper has been to present in a succinct form the variable coefficient $\mathrm{KdV}$ equation which describes the propagation of internal solitary waves in a variable current, and to obtain some relatively simple, albeit approximate, formulae for the variation of the solitary wave amplitude due to the variation in the basic current. The general theory was presented in section 1, based on the theory described by Grimshaw (1979, 1981). For the sake of completeness we have included in the Appendix a derivation of the variable coefficient $\mathrm{KdV}$ equation in the one-dimensional 
case when the solitary wave and the current are both directed in a single direction.

Then in section 2 we have considered two cases when the basic current contains vertical shear, but with suitable simplifications and approximations it is possible to obtain explicit formulae for the variation of the solitary wave amplitude due to horizontal variability in the basic current. Although the formulae we have derived hold under restrictions which may not always be met in practice, we hope that the formulae will be useful in providing at least a qualitative guide to the effect of variable current shear on internal solitary waves. To obtain results for more general basic currents would seem to require numerical integration of the modal equations ( $3 a-c)$, and numerical evaluation of the coefficients (eqn. $(6 a-c)$ ). Although this is always possible in any given practical case, we have not chosen that course here, as our aim has been to obtain useful analytical results. In section 3 we have presented some results for internal solitary waves propagating through geostrophic currents, tidal flows or inertial waves, under the assumption that the basic current is depth-independent. This last hypothesis allowed us to obtain an explicit formula (eqn. 45) for the variation of the solitary wave amplitude, which shows that the variation is entirely due to the spreading of the rays associated with the basic current. Three special cases were looked at in detail.

\section{ACKNOWLEDGEMENT}

This work was initiated while $\mathrm{X}$. Zhou was a visitor in the School of Mathematics University of N.S.W. He wishes to thank the School of Mathematics for its hospitality and to acknowledge the support of the grants from the Australian Department of Science, the National Nature Science Fund Committee of China and the Chinese Academy of Sciences. The work was completed while R. Grimshaw was a visitor to the Institute of Mechanics, Chinese Academy of Sciences, and he is grateful for their hospitality on that occasion.

\section{APPENDIX}

Derivation of the evolution equation in the one-dimensional case for a steady basic flow

When the basic flow is independent of $Y^{\prime}$ and $T^{\prime}$, and is directed in the $X^{\prime}$ direction, and the solitary waves also propagate in the $X^{\prime}$ direction, the ray equations ( $4 a$ and $b$ ) are readily solved and the evolution equation (eqn. (5)) simplifies to eqn. (13a). This reduction was described at the end of 
section 1. However, since the derivation of the variable coefficient $\mathrm{KdV}$ equation in the general case (Grimshaw, 1981) is rather abstract and lengthy, we present in this Appendix a brief account of a more direct derivation for this special case. Note that in the title of this Appendix we have called this the one-dimensional case because the basic flow has only one horizontal component which points in the $X^{\prime}$ direction, although, of course, the total flow is two-dimensional.

Let the steady basic flow be described by the horizontal velocity $\mathbf{u}_{0}=$ $\left(u_{0}\left(X^{\prime}, z\right), 0\right)$, a vertical velocity $\epsilon^{3} w_{0}\left(X^{\prime}, z\right)$, the density $\rho_{0}\left(X^{\prime}, z\right)$ and the pressure $p_{0}\left(X^{\prime}, z\right)$. They satisfy the Euler equations for inviscid, incompressible flow

$\rho_{0}\left(u_{0} u_{0 X^{\prime}}+w_{0} u_{0 z}\right)+\sigma^{-1} p_{0 X^{\prime}}=F_{0}$

$\epsilon^{6} \rho_{0}\left(u_{0} w_{0 X^{\prime}}+w_{0} w_{0 z}\right)+\sigma^{-1}\left(p_{0 z}+\rho_{0}\right)=\epsilon^{6} G_{0}$

$u_{0 X^{\prime}}+w_{0 z}=0$

$u_{0} \rho_{0 X^{\prime}}+w_{0} \rho_{0 z}=0$

Here $F_{0}$ and $G_{0}$ are body forces which allow us to specify the velocity field $\left(u_{0}, w_{0}\right)$ arbitrarily subject only to the kinematic conditions in eqns. 14a and $14 \mathrm{~b} . F_{0}$ and $G_{0}$ may be external force fields, or internal friction forces, but in either case describe non-conservative effects. The boundary conditions at the free surface $z=\zeta_{0}\left(X^{\prime}\right)$ and the rigid bottom $z=-h\left(X^{\prime}\right)$ are

$w_{0}-u_{0} \zeta_{0 X^{\prime}}=0, \quad$ or $z=\zeta_{0}\left(X^{\prime}\right)$

$p_{0}=0, \quad$ or $z=\zeta_{0}\left(X^{\prime}\right)$

$w_{0}+u_{0} h_{X^{\prime}}=0, \quad$ or $z=-h\left(X^{\prime}\right)$

Next let the wave field be described by a horizontal velocity $u$, a vertical velocity $w$, a density $\rho$ and a pressure $p$. Then the total field $u_{0}+u$, etc., satisfies the Euler equations for inviscid, incompressible flow. Subtracting the basic flow equations (A.1a-d) we find that

$$
\begin{aligned}
& \left(\rho_{0}+\rho\right)\left[u_{t}+\left(u_{0}+u\right) u_{x}+\left(\epsilon^{3} w_{0}+w\right) u_{z}+w u_{0 z}+\epsilon^{3} u u_{0 X^{\prime}}\right] \\
& \quad+\epsilon^{3} \rho\left(u_{0} u_{0 X^{\prime}}+w_{0} u_{0 z}\right)+\sigma^{-1} P_{x}=0 \\
& \left(\rho_{0}+\rho\right)\left[w_{t}+\left(u_{0}+u\right) w_{x}+\left(\epsilon_{0}^{3}+w\right) w_{z}+\epsilon^{3} w w_{0 z}+\epsilon^{6} u w_{0 X^{\prime}}\right] \\
& \quad+\epsilon^{6} \rho\left(u_{0} w_{0 X^{\prime}}+w_{0} w_{0 z}\right)+\sigma^{-1}\left(p_{z}+\rho\right)=0 \\
& u_{x}+w_{z}=0 \\
& \rho_{t}+\left(u_{0}+u\right) \rho_{x}+\left(\epsilon^{3} w_{0}+w\right) \rho_{z}+w \rho_{0 z}+\epsilon^{3} u \rho_{0 X^{\prime}}=0
\end{aligned}
$$


The boundary conditions are

$\zeta_{t}+\left(u_{0}+u\right)\left(\zeta_{0}+\zeta\right)_{x}-\left(\epsilon^{3} w_{0}+w\right)=0, \quad$ on $z=\zeta_{0}+\zeta$

$p_{0}+p=0$, on $z=\zeta_{0}+\zeta$

$w+\epsilon^{3} u h_{X^{\prime}}=0, \quad$ on $z=-h$

Here $\zeta$ is the displacement of the free surface due to the waves.

To describe weakly non-linear longwaves, we let $W\left(X^{\prime}\right)$ be the speed of linear longwaves, and put

$\theta=\epsilon^{-2}\left(s-T^{\prime}\right), \quad s=\int_{0}^{X^{\prime}} W^{-1} \mathrm{~d} X^{\prime}$.

Thus $\theta$ is a phase variable for linear longwaves and scales with $\epsilon$, while $s$ is a time-like variable which scales with $\epsilon^{3}$, where we recall from eqn. (1) that $X^{\prime}$ and $T^{\prime}$ scale with $\epsilon^{3}$. Then we let the wave field depend on $\theta, s$ and $z$, so that $u=u(\theta, s ; z)$, etc. Next we recall that the wave field scales with $\epsilon^{2}$, and put

$\left[\begin{array}{l}u \\ w \\ p \\ \rho\end{array}\right]=\epsilon^{2}\left[\begin{array}{c}u_{1} \\ \epsilon w_{1} \\ \sigma p_{1} \\ \sigma p_{1}\end{array}\right]+\epsilon^{4}\left[\begin{array}{c}u_{2} \\ \epsilon w_{2} \\ \sigma p_{2} \\ \sigma p_{2}\end{array}\right]+\ldots$

On substituting into eqn. (A.3a-d) we find that, to leading order,

$\rho_{0}\left(-\bar{W} u_{1 \theta}-W \bar{W}_{2} w_{1}\right)+p_{1 \theta}=0$

$p_{1 z}+\rho_{1}=0$

$u_{1 \theta}+W w_{1 z}=0$

$-\bar{W} \rho_{1 \theta}-W \rho_{0} N^{2} w_{1}=0$

where

$\bar{W}=w-u_{0}$

Similarly we find that the boundary conditions (A.4a-c) become, to leading order,

$-\bar{W} \zeta_{1 \theta}-W w_{1}=0, \quad$ on $z=\zeta_{0}$

$\sigma p_{1}-\rho_{0} \zeta_{1}=0, \quad$ on $z=\zeta_{0}$

$w_{1}=0, \quad$ on $z=-h$ 
The solution of eqns. (A.7a-d) and boundary conditions $(\mathrm{A} .8 \mathrm{a}-\mathrm{c})$ is given by

$$
\left[\begin{array}{c}
u_{1} \\
w_{1} \\
p_{1} \\
\rho_{1}
\end{array}\right]=\left[\begin{array}{c}
A(\bar{W} \phi)_{z} \\
-A \theta W^{-1} \bar{W} \phi \\
A \rho_{0} \bar{W}^{2} \phi_{z} \\
A \rho_{0} N^{2} \phi
\end{array}\right]
$$

where the modal function $\phi(s ; z)$ is defined by eqn. (3a-c) and now $\bar{W}=W-u_{0}$ (see eqn. (3d), where here $l=0$ ). The modal equations ( $\left.3 \mathrm{a}-\mathrm{c}\right)$ determine both $\phi(s ; z)$ and the linear longwave phase speed $W$. Also, we recall that $A(s, \theta)$ is defined so that to leading order the vertical particle displacement is $\epsilon^{2} A \phi$.

At the next order we find from eqn. (A.3a-d) that

$$
\begin{aligned}
& \rho_{0}\left(-\bar{W} u_{2 \theta}-W \bar{W}_{z} w_{2}\right)+p_{2 \theta}=F_{2} \\
& p_{2 z}+\rho_{2}=\rho_{0} W^{-1} \bar{W} w_{1 \theta} \\
& u_{2 \theta}+W w_{2 z}=-u_{1 s} \\
& -\bar{W} \rho_{2 \theta}-W \rho_{0} N^{2} w_{2}=G_{2}
\end{aligned}
$$

where

$$
\begin{array}{lr}
F_{2}=-\rho_{0}\left[\left(u_{0} u_{1}\right)_{s}+u_{1} u_{1 \theta}+W\left(w_{0}+w\right) u_{1 z}\right]-p_{1 s}+\frac{\rho_{1}}{\rho_{0}}\left(\sigma p_{1 \theta}+p_{0 s}-\sigma W F_{0}\right) \\
G_{2}=-\left(u_{0} \rho_{1 s}+u_{1 \rho 0 s}+u_{1} \rho_{1 \theta}\right)+W\left(w_{0}+w_{1}\right) \rho_{1 z} & \text { (A.10e) }
\end{array}
$$

Similarly, we find that the boundary conditions are

$$
\begin{aligned}
& -\bar{W} \zeta_{2 \theta}-W w_{2}=H_{2}, \quad \text { on } z=\zeta_{0} \\
& \sigma p_{2}-\rho_{0} \zeta_{2}=\sigma \rho_{1} \zeta_{1}-\frac{1}{2} \sigma \rho_{0} N^{2} \zeta_{1}^{2}, \quad \text { on } z=\zeta_{0} \\
& w_{2}=-W^{-1} u_{1} h_{s}, \quad \text { on } z=-h
\end{aligned}
$$

where

$$
H_{2}=-\left[\left(u_{0} \zeta_{1}\right)_{s}+\left(u_{1} \zeta_{1}\right)_{\theta}+u_{0 z} \zeta_{1} \zeta_{1 \theta}+u_{1} \zeta_{0 s}+u_{0 z} \zeta_{0 s} \zeta_{1}\right]
$$

Putting $W w_{2}=-\bar{W} \phi_{2}$ and eliminating $u_{2}, p_{2}$ and $\rho_{2}$ from eqn. (A.10a-d) we find that

$$
\left(\rho_{0} \bar{W}^{2} \phi_{2 z}\right)_{z}+\rho_{0} N^{2} \phi_{2}=I_{2}
$$

where

$$
I_{2}=-F_{2 z}+\bar{W}^{-1} G_{2}+\left(\rho_{0} \bar{W} u_{1 s}\right)_{z}+\rho_{0} \bar{W} W^{-1} w_{1 \theta \theta}
$$


The boundary conditions $(\mathrm{A} .11 \mathrm{a}-\mathrm{c})$ become

$\sigma \rho_{0} \bar{W}^{2} \phi_{2 z}-\rho_{0} \phi_{2}=J_{2}$, on $z=\zeta_{0}$

where

$J_{2}=-\bar{W}^{-1} \rho_{0} H_{2}-\sigma F_{2}+\sigma \rho_{0} \bar{W} u_{1 s}+\sigma\left(\rho_{1} \zeta_{1}\right)_{\theta}-\sigma \rho_{0} N^{2} \zeta_{1} \zeta_{1 \theta}$

$\bar{W} \phi_{2}=u_{1} h_{s}$, on $z=-h$

Equation (A.12a) with the boundary conditions (A.13a and c) has a solution if and only if the following compatibility condition is satisfied

$\int_{-h}^{\zeta_{0}} I_{2} \phi \mathrm{d} z-\left(\bar{W}^{2} \phi_{z} J_{2}\right)_{\zeta_{0}}-\left(\rho_{0} \bar{W} \phi_{z} u_{1} h_{s}\right)_{-h}=0$

This is established either by constructing the general solution of eqn. (A.12a) using the method of variation of parameters, or by finding the adjoint operator to the left-hand side of eqn. (27a) and then applying the Fredholm alternative condition. After substitution of the expressions (A.9), the condition (A.14) is seen to be an equation for $A(s, \theta)$. Upon simplification it may be shown to reduce the $\mathrm{KdV}$ equation (13a), with the coefficients given by eqns. (6a-c) and (7), where we note that with $l=0, \kappa=W^{-1}, V_{1}=W$, $V_{2}=0$, and hence $s$ and $\theta\left(=\epsilon^{-2} \Theta\right)$ as defined by eqns. (11a and b) and (12) agree with eqn. (A.5). Also we now find that

$I \gamma=-\int_{-h}^{\zeta_{0}} \phi \phi_{z} F_{0 z} \mathrm{~d} z$

\section{REFERENCES}

Apel, J.R., 1980. Satellite sensing of ocean surface dynamics, Ann. Rev. Earth Planet. Sci., 8: $303-342$.

Benney, D.J., 1966. Long non-linear waves in fluid flows. J. Math. Phys., 45: 52-63.

Chang, P., Melville, W.K. and Miles, J.W., 1979. On the evolution of a solitary wave in a gradually varying channel. J. Fluid Mech., 95: 401-414.

Christie, D.R., Muirhead, K.J. and Hales, A.L., 1978. On solitary waves in the atmosphere. J. Atmos. Sci., 35: 805-825.

Clarke, R.H., Smith, R.K. and Reid, D.G., 1981. The Morning Glory of the Gulf of Carpentaria: an atmospheric undular base. Mont. Weather Rev., 109: 1726-1750.

Djordjevic, V.D. and Redekopp, L.G., 1978. The fission and disintegration of internal solitary waves moving over two-dimensional topography. J. Phys. Ocean., 8: 1016-1024.

Farmer, D.M., 1978. Observations of long nonlinear internal waves in a lake. J. Phys. Ocean., 8: 63-73.

Gill, A.E., 1982. Atmosphere-Ocean Dynamics, Academic Press, New York, 662 pp.

Grimshaw, R., 1979. Slowly varying solitary waves. I. Korteweg-de Vries equation. Proc. R. Soc., London, Ser. A, 368: 359-375.

Grimshaw, R., 1981. Evolution equations for nonlinear internal waves in stratified shear flows. Stud. Appl. Math., 65: 159-188. 
Grimshaw, R., 1983. Solitary waves in density stratified fluids. In: V. Nigul and J. Engelbrecht (Editors), Nonlinear Deformation Waves. Proceedings of the IUTAM Symposium, Tallinn, pp. 431-447.

Grimshaw, R., 1986. Theory of solitary waves in shallow fluids. In: Encyclopedia of Fluid Mechanics. Vol. 2. Gulf Publishing Co., pp. 3-25.

Johnson, R.S., 1973. On the development of a solitary wave moving over an uneven bottom. Proc. Camb. Philos. Soc., 73: 183-205.

Joshi, N., 1987. Painleve property of a general variable-coefficient Korteweg-de Vries equation (preprint).

Kakutani, T., 1971. Effect of an uneven bottom on gravity waves. J. Phys. Soc. Japan, 30: $272-276$.

Knickerbocker, C.J. and Newell, A.C., 1980. Shelves and the Korteweg-de Vries equation. J. Fluid Mech., 98: 803-818.

Long, R.R., 1953. Some aspects of the flow of stratified fluids. I. A theoretical investigation. Tellus, 5: 42-57.

Maslowe, S.A. and Redekopp, L.G., 1980. Long nonlinear waves in stratified shear flows. J. Fluid Mech., 101: 321-348.

Miles, J.W., 1980. Solitary waves. Ann. Fluid Mech., 12: 11 43 .

Noonan, J.A. and Smith, R.K., 1985. Linear and weakly nonlinear internal wave theories applied to "Morning Glory" waves. Geophys. Astrophys. Fluid Dyn., 33: 123-143.

Ostrovsky, L.A. and Pelinovsky, E.N., 1970. Transformation of surface waves in fluids of variable depth. Izv. Acad. Sci. USSR, Atmos. Ocean. Phys., 9: 934-939.

Smyth, N. and Holloway, P., 1987. Hydraulic jump and undular bore formation on a shelf break (preprint).

Zhou, X., 1985. Effect of current on the fission of a solitary wave. Sci. Sinica, Ser. A, 28: $1278-1290$.

Zhou, X., 1987. The fission of a solitary wave in a weakly stratified fluid. In: Proceedings of the International Conference on Fluid Mechanics, Beijing, pp. 459-464. 